\title{
Route Optimization in Network Mobility: Solutions, Classification, Comparison, and Future Research Directions
}

\author{
Abu Zafar M. Shahriar, Mohammed Atiquzzaman, Senior Member, IEEE, and William Ivancic
}

\begin{abstract}
NEtwork MObility (NEMO) handles mobility of a set of mobile nodes in an aggregate way using one or more mobile routers. NEMO introduces several advantages, such as reduced signaling, increased manageability, reduced power consumption and conservation of bandwidth when compared to individual host mobility. NEMO Basic Support Protocol (BSP), the IETF standard for NEMO, suffers from a number of limitations, like inefficient route and increased handoff latency. Most of the recent research efforts on NEMO have concentrated on solving the problem of inefficient route resulting in several route optimization schemes to solve the problem. To choose a route optimization scheme, it is very important to have a quantitative comparison of the available route optimization schemes. The objective of this article is to survey, classify and compare the route optimization schemes proposed in the literature over the last five years. We classify the schemes based on the basic approach for route optimization, and compare the schemes based on protocol overhead, such as header overhead, amount of signalling, and memory requirements. We conclude that performance of the classes of schemes has to be evaluated under criteria such as available bandwidth, topology of the mobile network and mobility type.
\end{abstract}

Index Terms-Network Mobility, Basic Support Protocol, Inefficient Route, Route Optimization.

\section{INTRODUCTION}

W IRELESS connectivity gave rise to the demand for ubiquitous connectivity of both static and mobile IPenabled devices. In the future, it may be common for several devices which are connected in a Local Area Network to move together. Examples include devices on vehicles connected to on-board Internet-connected LAN, a person carrying several devices which are connected to a Personal Area Network (PAN), etc.

Existing Internet is not designed to handle mobility due to IP's location-based addressing scheme where IP addresses are tied to geographical areas. A host moving between networks in different geographical areas needs to obtain a new IP address, and therefore, communication may become inefficient while maintaining reachability and session continuity. To overcome the inefficiency of current IP addressing, Internet Engineering

Manuscript received; revised. The research reported in this article was funded by NASA Grant NNX06AE44G.

A. Z. M. Shahriar and M. Atiquzzaman are with the Telecommunications and Networks Research Lab, School of Computer Science, University of Oklahoma, Norman, OK 73019-6151 USA (e-mail: shahriar,atiq@ou.edu).

W. Ivancic is with the Satellite Networks and Architectures Branch, NASA Glenn Research Center, Cleveland, OH 44135 (e-mail: wivancic@grc.nasa.gov).

Digital Object Identifier 10.1109/SURV.2010.1001xx.
Task Force (IETF) designed solutions such as Mobile IP (MIP) [1] and MIPv6 [2] to support mobility of a host. A summary of some of the host mobility protocols (including MIP and MIPv6) can be found in [3].

Managing mobility of a number of devices in a moving LAN or PAN using host mobility protocols for each device increases signaling overhead during handoff, power consumption and manageability. Moreover, not all the hosts in the mobile network may be sophisticated enough to support mobility protocols or have powerful transceivers to communicate with different access points. IETF developed NEtwork MObility (NEMO) where one or more routers, called mobile routers, manage the mobility of all the hosts in a network. NEMO supports nested mobile network, where a mobile network is connected under another mobile network. Multiple levels of nesting happens when a number of mobile networks are connected in a hierarchy.

IETF extended MIPv6 to design NEMO Basic Support Protocol (NEMO BSP) [4] to handle network mobility, where hosts in a mobile network are reachable through a home agent. Packets to and from the mobile network travel through a bidirectional tunnel [5] between a home agent and a mobile router. Tunneling packets through the home agent results in the problems of inefficient route and increased header overhead due to encapsulation. The problem intensifies with multiple levels of nesting giving rise to multiple encapsulation of packets which travel through multiple home agents. Inefficient routes also result in large handoff latencies.

Inefficient route increases end-to-end delay, resulting in performance degradation of real-time and acknowledgementbased data transfers. The problem increases further with increase of nesting level. Header overhead results in bandwidth inefficiency, and increases the possibility of fragmentation. Consequently, most of the research efforts on NEMO concentrate on Route Optimization (RO) i.e. solving the problem of inefficient route and header overhead. Our goal in this paper is to survey the state-of-the-art in RO over the last five years.

The basic principle of RO is to enable packets to directly reach the mobile network by avoiding multiple tunnels through home agents. RO, however, requires addressing the following challenges:

- How can packets reach the foreign network to which the mobile network is connected?

- How can packets be routed inside the mobile network?

Apart from the above two challenges, there is another chal- 
lenge of how to route packets between two hosts inside a mobile network without traversing the home agent. Several RO schemes that address the above three challenges have been proposed in the literature. Perera et al. [6] provide a comprehensive introduction to NEMO, advantages and limitations of NEMO BSP, a review of the RO schemes and propose future research directions for NEMO. Being an emerging area of research, there have been additional work [7]-[12], [12]-[32] on NEMO RO than those reported in [6].

Addressing the challenges of RO raises issues, such as additional header, signaling and memory requirement. Therefore, RO schemes differ in their approaches to solve the inefficient route problem while trading off the issues. Lim et al. classified the RO schemes based on their approaches [33]. However, like [6], the classification does not include many recently proposed schemes. In this paper, our objective is to provide a comprehensive up-to-date summary of the RO schemes, and classify and compare the schemes (like in [33]). Unlike that in [33], we also provide qualitative comparison among the schemes in each class.

Our contribution is to identify the strategies adopted by the RO schemes, followed by classification of the schemes based on the strategies. We introduce a new class of schemes based on the the RO techniques proposed in [28], [30], [31]. These RO techniques are fundamentally different from the other class of schemes reported in [33] in terms of directly using the routing protocol in the Internet. Our classification enables hierarchical as well as individual comparison of the schemes. We also reveal degree of RO, deployability and type of RO supported by each class in general. The comparison shows the differences among the schemes in terms of issues, such as additional header, signaling and memory requirement. This comparison implies the need for performance evaluation of the schemes for suitability under conditions such as availability of bandwidth, resource constraints (such as memory constraints), and mobility patterns. Future research on $R O$ can use the results in this paper by building on and being more focused by using the comparison presented in this paper.

The rest of the paper is organized as follows. Sec. II presents the NEMO architecture, NEMO BSP protocol, and limitations of the protocol. Challenges to be addressed for RO, issues in $\mathrm{RO}$, and summary and classification of the RO schemes based on the strategies used are presented in Sec. III. Future research directions are highlighted in Sec. IV, followed by concluding remarks in Sec. V.

\section{NEMO}

In this section, we describe the NEMO architecture and NEMO BSP along with its limitations.

\section{A. Architecture}

Fig. 1 shows the NEMO architecture where one or more routers, called Mobile Router (MR) (like TLMR and MR1), act as gateways for the Mobile Network Nodes (MNNs) [34]. When the point of attachment of a mobile network changes due to mobility, MRs perform handoff to keep the movement transparent to MNNs. The following lists the possible types of MNNs:

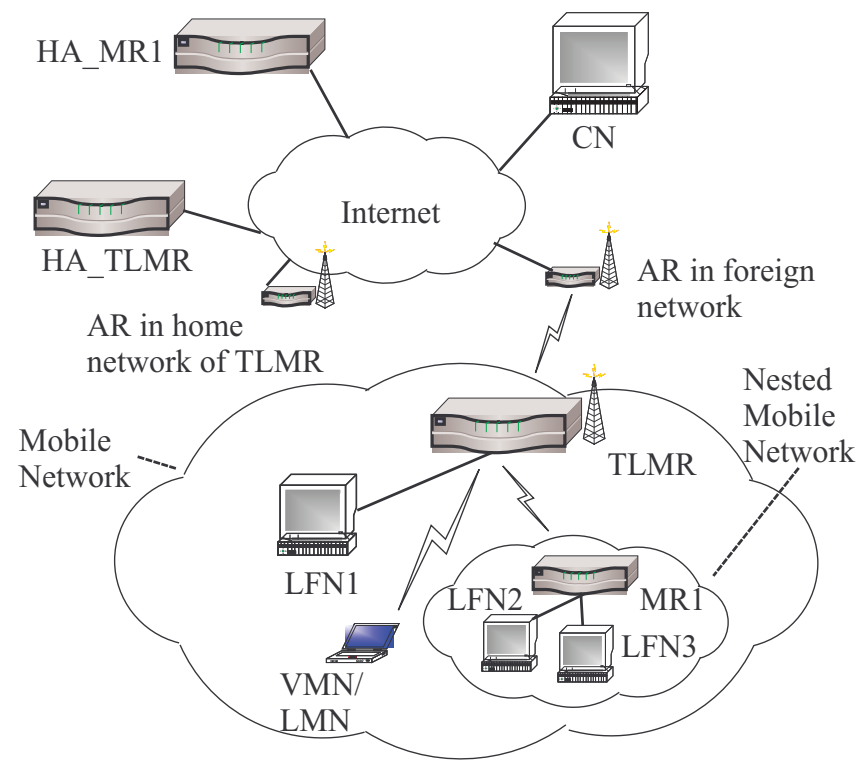

Fig. 1. Architecture of NEMO showing one level of nesting.

- Local Fixed Node (LFN): These nodes do not move with respect to the mobile network.

- Local Mobile Node (LMN): These nodes usually reside in the mobile network but can move to other networks.

- Visiting Mobile Node (VMN): These nodes belong to another network but is currently attached to the mobile network.

- MR: An MNN can act as an MR to form a nested mobile network.

LMNs, VMNs and MRs implement mobility protocols; we will refer to these nodes as Mobility Capable Nodes (MCNs).

An MR attaches to another MR to form a nested mobile network. A number of MRs connected in series can result in multiple levels of nesting. A Top Level MR (TLMR) attaches directly to the wired network through Access Routers (ARs). In Fig. 1, the mobile network under MR1 is nested under TLMR's mobile network; MR1's mobile network thus has a nesting level of one.

The network to which a mobile network is usually connected is called the home network. An MR is registered with a router, called Home Agent (HA), in its home network. In Fig. 1, HA_TLMR and HA_MR1 are the HAs for the mobile routers TLMR and MR1, respectively. A node that communicates with MNNs is called Correspondent Node $(\mathrm{CN})$ which can also be an MNN. CNs and MNNs communicate using NEMO BSP as described in Sec. II-B.

\section{B. NEMO BSP}

The home network delegates one or more prefixes to the TLMR for use by its MNNs. TLMR has a Home Address (HoA) through which it is reachable in its home network. When the TLMR moves to a foreign network (any network other than home network), it obtains a new address (called Care-of-Address (CoA)) from the foreign network and registers the CoA (and optionally the delegated prefixes) with the HA_TLMR by sending a Binding Update (BU). The BU contains a Mobile Router Flag indicating that TLMR is acting 


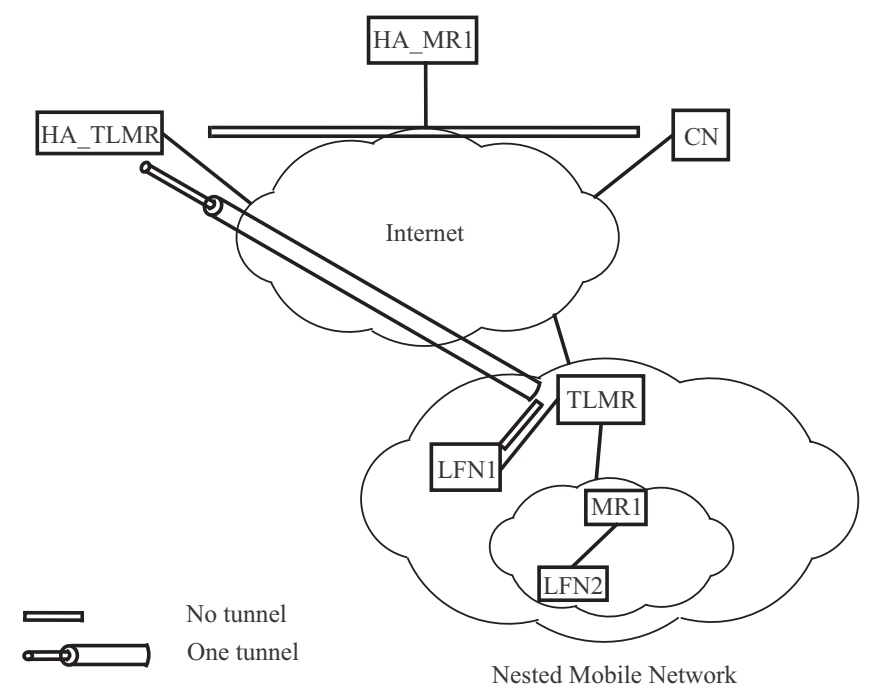

Fig. 2. Single tunneling when routing packet for LFN1.

as a router. In response to the BU, HA_TLMR sends a positive Binding Acknowledgement (BA) to indicate that forwarding to TLMR is set, and creates a binding cache entry that maps the TLMR's HoA and prefixes to the CoA of the TLMR. A bidirectional tunnel [5], established between HA_TLMR and TLMR, tunnels all packets between CN and MNN. Note that the above registration process is similar to that of MIPv6 registration [2] except setting of the Mobile Router Flag and sending of prefix in the BU.

A nested mobile network is created when MR1 moves under TLMR. MR1 obtains a CoA from TLMR's prefix followed by registration of MR1 with HA_MR1 and tunnel setup, which is similar to the registration of TLMR as described above.

Fig. 2 shows the routing of packets from CN to LFN1. Since LFN1 obtains its address from TLMR's prefix (delegated by TLMR's home network), the packet is routed towards the HA_TLMR. HA_TLMR encapsulates and forwards the packet to TLMR. TLMR receives, decapsulates and forwards the packet to LFN1. Packets in the reverse direction take the same path in reverse under going encapsulation and decapsulation at TLMR and HA_TLMR, respectively.

Fig. 3 shows packets going from $\mathrm{CN}$ to LFN2 through multiple tunnels in a nested mobile network. Since LFN2 obtains its address from MR1's prefix (delegated by MR1's home network), the packets are intercepted by HA_MR1, encapsulated and tunneled to MR1. Since MR1's CoA is obtained from TLMR's prefix, the packets are intercepted by HA_TLMR, encapsulated further and tunneled to TLMR, resulting in multiple encapsulations. Encapsulated packets, on reaching TLMR, are decapsulated and forwarded to MR1, which again decapsulates the packets and forwards them to LFN2.

Although NEMO BSP provides aggregate mobility management of a group of nodes resulting in advantages mentioned earlier in Sec. I, advantages are not without limitations. Limitations of NEMO BSP are described in Sec. II-C.

\section{Limitations of NEMO BSP}

NEMO BSP is an extension to MIPv6 to allow an MIPv6

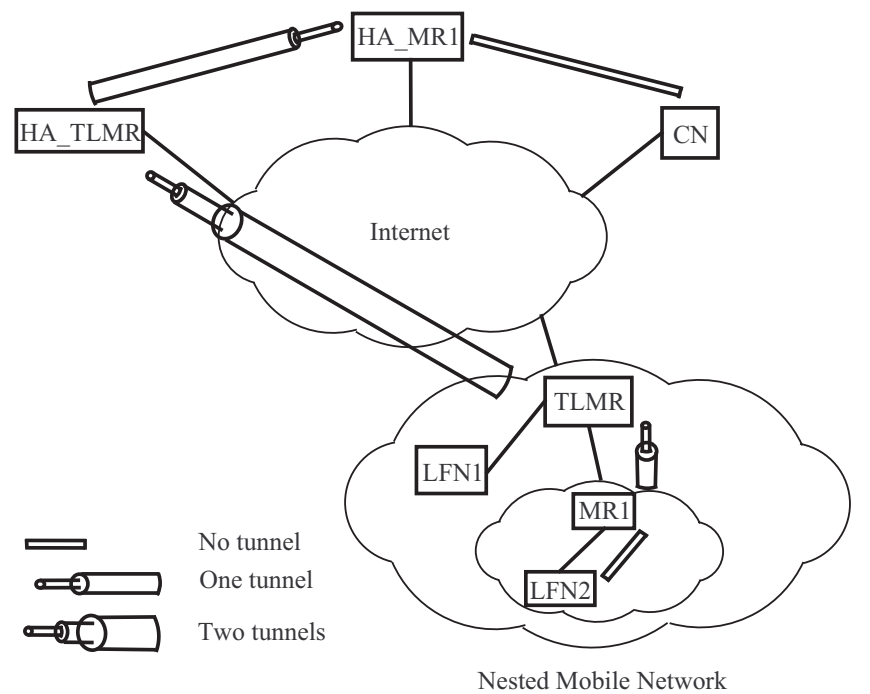

Fig. 3. Multiple tunneling in nested mobile network.

node to act as a router with address delegation capability. Therefore, NEMO BSP inherits the limitations of MIPv6 as well as having its own limitations that are described below.

1) Suboptimal Route: As it is evident from Fig 3, packets sent by $\mathrm{CNs}$ reach the mobile network through one or more (due to nesting) bi-directional tunnels between the HA and the MR. Thus, the route traversed by packets may be suboptimal when the mobile network and $\mathrm{CN}$ are in the same network (or topologically close) that is far away from the HA. Suboptimal route results in inefficiencies such as higher end-to-end delay, additional load on infrastructure, susceptibility to link failures etc. that are presented in detail in [35]. Moreover, requirement of all packets from or to the mobile network to pass through HA creates bottleneck [35].

Header overhead is another problem associated with the problems of suboptimal route [35]. As a packet passes through each tunnel, it is encapsulated resulting in increased packet size. Encapsulation results in header overhead that decreases bandwidth efficiency, and increases the chance of fragmentation. Moreover, encapsulated packets are also decapsulated as many times as the number of encapsulations. Encapsulation and decapsulation require additional processing at $\mathrm{HA}$ and MR.

2) Handoff delay: Handover of an MR is similar to that of an MIPv6 node [2]. When an MR hands off from one network to another, it has to discover an access router to obtain a CoA, and perform registration with the HA. This handoff procedure results in delay that interrupts ongoing connections. Problems of suboptimal route, discussed in Sec. II-C1, reinforces the delay.

The problem of reducing handoff delay is not unique to NEMO, and has been adequately addressed for MIPv6. The problems of suboptimal route are aggravated when nesting (unique to NEMO) occurs; therefore, RO is an active area of research in NEMO. In Sec. III, we present RO challenges, issues and the state-of-the-art RO schemes. 


\section{Route Optimization (RO)}

$\mathrm{RO}$ requires addressing several challenges which raises issues that affect performance and applicability of the schemes. To tradeoff the gain of RO with the performance and applicability, several schemes have been proposed. In this section, we present the RO schemes and their challenges in and issues.

\section{A. Challenges in RO}

$\mathrm{RO}$ requires bypassing the HAs when packets are sent between $\mathrm{CN}$ and MNNs. Bypassing HAs gives rise to the following two major challenges which have to be addressed by RO schemes:

- How can a packet destined to an MNN reach the TLMR attached to the foreign network to which the MNN is attached (directly or indirectly)?

- How is a packet routed inside the mobile network after reaching TLMR?

The challenges are addressed by majority of the RO schemes that focus on optimizing route between a $\mathrm{CN}$ in wired network and an MNN. Addressing above mentioned challenges is not sufficient for optimizing route between two MNNs (intra mobile network communication [35]) that involves more encapsulation/decapsulation, and longer route than those required in transmission of packets between a $\mathrm{CN}$ in the wired network network and an MNN. The challenge of RO in intra mobile network case (Intra RO), is how to route packets between two MNNs without letting the packet outside the mobile network; some RO schemes also address this later challenge. Although our focus is on RO between $\mathrm{CN}$ and MNNs, the schemes that perform Intra RO are included because they also optimize route for communication between a $\mathrm{CN}$ and an MNN. We report whether a scheme perform Intra RO as we introduce the schemes.

\section{B. Issues in $R O$}

Addressing the challenges mentioned in Sec. III-A raises several issues that were reported in [36] addition to header overhead (II-C) and Intra RO (III-A) issues as given below.

1) Signaling: When a mobile network moves, only the MR to which the movement is visible needs to perform signaling with its HA. RO schemes may require more signaling than NEMO BSP to convey prefix of foreign network to $\mathrm{CN}$, and to expose the route from TLMR to MNN. Signaling packets competes with data packets for bandwidth not only inside the mobile network but also in the Internet.

2) Memory requirement: Schemes have to maintain various state information regarding the route and CN-MNN pairs. Maintaining state information requires memory that can be a limiting factor in memory constrained environments involving small devices, for example, small sensors and PDAs.

3) Degree of RO: In an effort to tradeoff issues, such as signaling, some schemes allow one or two levels of tunneling or some non-optimality in the route between a $\mathrm{CN}$ and an MNN. Although the level of tunneling or the extent of non-optimality is small, it gives rise to the inefficiencies like high end-to-end delay, load on infrastructure, increased susceptibility to link failures, and header overhead discussed in Sec. II-C1. To characterize the degree of RO, we use the term optimal and near optimal for routes that are optimal and non-optimal (to some extent), respectively.

4) Header overhead: Header overhead is the additional information that are put into the header for RO. Header overhead consumes bandwidth and increases chance of fragmentation.

5) Intra RO: Route optimization between two MNNs within a mobile network is called Intra RO. With a focus on optimizing route between a $\mathrm{CN}$ and an $\mathrm{MNN}$, some of the schemes do not consider Intra RO.

6) Deployability: The schemes propose new functionalities for the existing hosts and routers in the Internet, for mobility entities such as MR, HA, and even propose new entities. Changes in mobility entities are tolerable because they are going to be introduced in the existing infrastructure if NEMO support is required. Changes in functionalities in hosts and routers in the existing infrastructure may not be easily applicable resulting in concern about deployability issue.

7) Location management: Location management is tracking the location of an MNN to ensure reachability and session continuity. In NEMO BSP and some RO schemes, location management is performed by HA. On the otherhand, some schemes propose location management by CNs, TLMRS, routers (closest to $\mathrm{CN}$ ) in the Internet, or by some new entities. Location management by HA, TLMR or new entities is easily deployable but susceptible to failure. Location management by CNs or routers in the Internet is less prone to failures because of no dedicated location manager is used; but requires changes in the existing routers and hosts raising deployability issue.

8) Location transparency: In NEMO BSP, MNNs except MRs, and CNs are transparent to location change. In an effort to optimize route, some of the schemes sacrifice location transparency resulting in requirement of mobility support from both MNNs and CNs. This makes the the schemes difficult to deploy due to required changes in functionality of the hosts in the Internet.

The eight issues mentioned above are used as criteria for comparison among the schemes in this paper. Additional criteria specific to a class are introduced later for comparison among the schemes.

\section{RO schemes}

To address the challenges mentioned in Sec. III-A, several RO schemes have been proposed in the literature. To trade off the issues mentioned in Sec. III-B, schemes follow various approach. Based on approach used, the various RO schemes that have been proposed can be generally classified as:

- Delegation

- Hierarchical

- Source routing

- BGP-assisted 


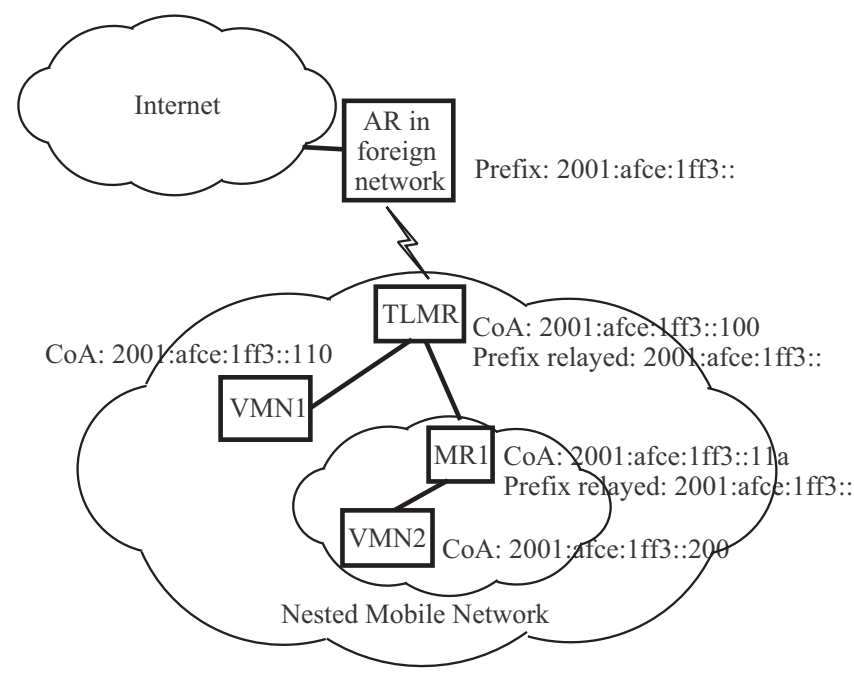

Fig. 4. Delegation approach for route optimization.

Based on the issues (see in Sec. III-B) over which the classes vary, Table I presents a comparative summary of the classes. In the rest of this section, we present the basic principle of each class, and a description and comparison of the schemes.

1) Delegation: In this class, prefix of the foreign network is delegated inside the mobile network. MCNs obtain CoAs from the prefix and send BUs to respective HAs and CNs. Therefore, any packet from $\mathrm{CN}$, addressed to CoA, reaches the foreign network without going through HAs. For example, as shown in Fig. 4, prefix 2001:afce:1ff3:: is relayed by TLMR inside its mobile network. VMN1 and MR1 obtains CoA 2001:afce:1ff3:110 and 2001:afce:1ff3:11a, respectively; and MR1, in turn, relays the prefix inside its network. Process of obtaining CoA from foreign network's prefix and packet routing inside the mobile network varies among the schemes in this class resulting in differences in signaling and memory requirement. A comparison based on the differences are presented in Table II.

The concept of prefix delegation is simple, and provides optimal route with low header overhead at the cost of sacrificing location transparency. Moreover, sending BU to $\mathrm{CN}$ requires additional signaling along with requirement of protocol support (location management along with HA) from $\mathrm{CN}$, making the schemes difficult to deploy. The schemes also do not focus on Intra RO.

a) Simple Prefix Delegation: In Simple Prefix Delegation, proposed by Lee et al. [37], a prefix that can be aggregated at the prefix of the foreign network is hierarchically delegated to the MRs. MRs advertise the delegated prefix inside its own network using Delegated Prefix Option in the header. Since prefix is hierarchically delegated, packet forwarding inside the mobile network can be done based on prefix of packets' destination address. This scheme, however, requires a prefix delegator in every mobile network, requiring additional overhead of performing extra functionality related to prefix delegation. Its signaling amount is proportional to number of MCNs, and in between low and high (i.e. medium) amount of signaling of other schemes in this class. Memory requirement is low because only attached MRs' prefix needs to be tracked as the next hop. The advantage of the signaling not being high costs incomplete RO for LFNs whereas route for MCNs is optimal.

The scheme, proposed by Mimoune et al. [7], is very similar to the Simple Prefix Delegation scheme [37] in terms of delegating prefix and obtaining CoA. Unlike the scheme proposed in [37] where CNs are updated by MCNs, MR notifies the border routers in home network about the delegated prefix. Border router makes an entry that maps home prefix to delegated prefix, and informs other border routers that eventually inform the $\mathrm{CN}$ about the prefix right after the communication has started. CN obtains the MNN's CoA obtained by combining its HoA and the prefix informed by the router, and can send packets using MNN's CoA. Although the scheme reduces signaing load which is distributed among border routers, functionalities of $\mathrm{CN}$ and routers need to be changed requiring change in infrastructure.

b) Neighbor Discovery Proxy (ND-Proxy): In this scheme, proposed by Jeong et al. [38], RO is achieved by advertising the prefix of the foreign network inside the mobile network. Each MR obtains a CoA from the advertised prefix and advertises the prefix inside its mobile network. All MCNs use the advertised prefix to obtain CoAs. Routing of packets is different from Simple prefix delegation (where prefix are hierarchically delegated) because all addresses are obtained from a single prefix. When TLMR receives a packet destined to an MNN, and the nexthop for the destination is not present in the routing table, it makes a neighbor discovery query. An MR attached below responds if the MNN's CoA that is being sought is directly under the MR. Otherwise, the MR relays the search message to MRs underneath, and replies to the query when an MR underneath responds with the CoA being sought. Thus, MRs actually act as proxy for MNNs for neighbor discovery.

Routing used in ND-Proxy will introduce delay at the start of communication. Yet, it has the advantage of not requiring a prefix delegator in every mobile network. Signaling requirement is similar to that of Simple prefix delegation whereas memory requirement is little higher (hence, low instead of lower) than Simple Prefix Delegation because of maintaining routing entries for all communicating MNNs underneath an MR.

In another scheme proposed by Song et al. [39], MRs advertise (like that in [38]) AR's prefix only (unlike [38]) to attached MRs that perform route optimization on behalf of attached MNNs (unlike [38]) by sending BU to corresponding CNs. Since MRs need to send BU to all $\mathrm{CNs}$ and track all $\mathrm{CNs}$, signaling and memory requirement for the scheme is high in this scheme.

c) Optimal routing for network mobility (Optinet):

Perera et al. [40] proposed an architecture called Optinet which is similar to Simple Prefix Delegation but with different prefix delegation procedure. Unlike Simple Prefix Delegation, a DHCP client in an MR obtains a prefix from the network it attaches to (a mobile network or a wired network). Petander et al. [41] extended Optinet (xOptinet) that reduces signaling by restricting the obtaining of the CoAs to only those nodes that are actively communicating with $\mathrm{CN}$ during handoff. Moreover, xOptinet optimizes the route for the LFNs by 
TABLE I

A COMPARISON AMONG DIFFERENT CLASSES

\begin{tabular}{|l|l|l|l|l|l|l|}
\hline Class & Degree of RO & Intra RO & Signaling & Header overhead & Deploybility & $\begin{array}{l}\text { Location } \\
\text { transparency }\end{array}$ \\
\hline Delegation & Optimal & No & High & Low & Difficult & No \\
\hline Hierarchical & Near optimal & Yes & Low & Medium & Easy & Yes \\
\hline Source routing & Optimal & No & High & High & Difficult & No \\
\hline BGP-assisted & Near optimal & Yes & Low & Low & Difficult & Yes \\
\hline
\end{tabular}

TABLE II

A COMPARISON OF THE SCHEMES IN DELEGATION CLASS SCHEMES

\begin{tabular}{|l|l|l|l|}
\hline Scheme & Signaling & Memory requirement & Other overheads \\
\hline Simple Prefix Delegation & Medium & Lower & Higher end-to-end delay for LFNs \\
\hline ND-Proxy & Medium & Low & Additional delay at the start of communication \\
\hline Optinet & High & High & None \\
\hline MIRON & High & High & None \\
\hline Ad hoc-based & Medium & Low & $\begin{array}{l}\text { Higher end-to-end delay for LFNs, Flooding of ad hoc } \\
\text { protocol messages }\end{array}$ \\
\hline OPR & Low & High & Per packet processing \\
\hline HIP-based & High & High & Per packet processing \\
\hline
\end{tabular}

having the MR perform RO signaling on behalf of attached LFNs. Unlike other schemes in Sec. III-C1, Optinet requires a DHCP client and a server at every mobile network. Moreover, LFNs' route optimization requires sending BUs to $\mathrm{CNs}$, and tracking LFN-CN communications resulting in high amount of signaling and memory requirement, respectively.

d) Mobile IPv6 Route Optimization for NEMO (MIRON): In MIRON, proposed by Calderon et al. [42], [43], like NDproxy, MCNs obtain the CoA from the prefix of the foreign access network. Upon attachment to an MR, an MCN obtains a CoA from MR's home prefix, and sends a BU to its HA. The MR intercepts the BU, and notifies the MCN to obtain a new CoA using PANA [44] and DHCP. MCN sends a DHCP request to obtain a $\mathrm{CoA}$. Instead of relaying the prefix (like ND-Proxy) inside the mobile network, this scheme relays the request to the DHCP server at the foreign network. Assigned $\mathrm{CoA}$ is then relayed back to the MCN. Relaying is performed by DHCP client and server component in MRs. After obtaining a CoA, MR notifies the attached MCNs to obtain a CoA. This procedure of obtaining a $\mathrm{CoA}$ is repeated at each handoff, and takes longer time than it takes in other schemes presented in Sec. III-C1. Like xOptinet, MIRON optimizes the route for the LFNs by having the MR perform RO signaling on behalf of attached LFNs, and hence signaling and memory requirement is high.

e) Ad hoc-based: In Ad hoc-based scheme proposed by $\mathrm{Su}$ et al. [8], like ND-Proxy, MCNs obtain CoA from the prefix of the foreign network. Unlike ND-proxy, for routing inside mobile network, the route between the $\mathrm{MNN}$ and the $\mathrm{AR}$ in foreign network is discovered using an Ad hoc protocol. Route discovery requires flooding of messages that consumes bandwidth as well as introduction of delay at the start of communication or after communication interrupted due to handoff. Moreover, Ad hoc network protocols are intended for unstable networks, and does not take advantage of the hierarchical nature of the nested mobile networks. Since it does not optimize route for LFNs, signaling requirement is similar to Simple Prefix Delegation at the cost of higher endto-end delay for LFNs' packets. Due to maintaining routing entry for all communicating MNNs, memory requirement is similar to ND-Proxy.

f) Optimal Path Registration (OPR): In OPR proposed by Park et al. [9], like ND-Proxy or Ad hoc-based scheme the prefix of the foreign network is advertised inside the mobile network. The difference of OPR with ND-Proxy and Ad hocbased schemes is that the prefix is relayed only to the MRs, resulting in movement transparency for other MNNs. To provide movement transparency, MRs translate prefix of source and destination addresses of outbound and inbound packets of its network. Movement transparency costs additional memory due to maintaining a translation table and processing cost per packet for address translation. Signaling is low in OPR than other schemes discussed in Sec. III-C1 because of not sending $\mathrm{BUs}$ to $\mathrm{CNs}$. To compensate for not sending $\mathrm{BU}, \mathrm{CNs}$ are informed of the change in translated address (like CoA in other schemes) by marking the packet's header. This costs the MR high memory due to state management to track every $\mathrm{CN}$ MNN communicating pair along with additional processing overhead per packet.

Kim et al. proposed a scheme that improves the performance of OPR by further reducing the number of BU [45]. To reduce $\mathrm{BU}$, the scheme proposed by $\mathrm{Kim}$ requires all $\mathrm{HAs}$ to join a multicast group that is managed by either AR or HA depending on when the multicast group is formed. For location update, AR sends a BU to the multicast group that reaches all HAs. However, this scheme requires ARs functionality to be modified for RO, and hence not easily deployable.

g) HIP-based: A Host Identification Protocol (HIP)based route optimization is proposed by Novaczki et al. [10], [11]. Like MRs in other schemes in delegation class, the mobile Rendezvous Servers (mRSVs) in HIP-NEMO obtain a prefix from the foreign network, and delegate parts of the prefix to attached mRSVs to advertise inside the mobile network which they are in. For route optimization, mRSV uses the prefix as location identifier of MNNs when sending location updates to CNs and RSVs (acts like HA and DNS), and translates the source/destination address of outgoing/incoming packets. When an mRSV attach to an AR, it obtains new prefix, performs location update signaling with CNs and RSVs on behalf of MNNs, and updates the prefix of the attached 
mRSVs that also do the same. Location update, attaching to a mobile network, and delegation of signaling to mRSVs are performed according to HIP [46]. Signaling for this scheme is the same as MIRON because of sending location updates to all CNs. Memory requirement and per packet processing overhead is like OPR because of similarity in address translation process. Although the entities that manage mobility functionalities such as location update, address mapping are entities defined in HIP, the basic approach is essentially the same as the other schemes in this class, and hence we include the scheme in this class.

2) Hierarchical: In the hierarchial class, a packet, rather than traveling through all HAs, reaches the foreign network either from MNN's HA (first HA) or traveling only through HA of MNN and TLMR. Unlike delegation-based approach, an MR does not send its CoA to CNs. Rather, an MR sends TLMR's CoA or HoA to HA. CNs use MNN's HoA to send packets to an MNN. Packets, sent by $\mathrm{CN}$ to $\mathrm{MNN}$, reach MNN's HA that tunnels the packets to TLMR's CoA or HoA. Packets, tunneled to CoA, directly reach the foreign network, where as packets, tunneled to HoA, reach TLMR's HA that tunnels packets to TLMR. On reaching TLMR, packets are routed to MNN by MRs that maintain a routing table containing the mapping of MNN's prefix to next hop MR. Fig. 5 shows an abstract view of the hierarchical class. TLMR_CoA is passed to HA_MR1 and HA_VMN by MR1 and VMN, respectively. Also, MR1 and VMN send their CoAs to TLMR to enable forwarding inside the mobile network. Therefore, a packet sent to VMN will first reach HA_VMN that tunnels the packet to the TLMR for forwarding towards the VMN. Thus, communication route is divided into two parts: the route between TLMR and HA_VMN, and the route from the TLMR to VMN. At least one tunnel always exists between the TLMR and HA_VMN. The route between CN and MR1 is similar to that between $\mathrm{CN}$ and VMN.

The schemes in this class mainly differ in the use of TLMR's CoA or HoA for tunneling, techniques to convey TLMR's address to MRs, and routing of packets inside mobile network resulting in differences in signaling, memory requirement and degree of RO. Moreover, depending on the use of HoA or CoA of the TLMR, the number of tunnels used for communication differs among the schemes; number of tunnels affects degree of RO and header overhead. In addition, location management entities also varies among the schemes. A comparative summary based on these differences are presented in Table III.

The schemes in this class require fewer number of signaling than delegation-based schemes because no BU is sent to CNs (except [18], [25], [46], [47]). This also makes CNs transparent to the mobility of communicating MNNs, yielding location transparency and easy deployability. Additionally, no $\mathrm{BU}$ is sent to HA for intra mobile network movement because of unchanged TLMR address, resulting in reduced signaling; this resembles Hierarchical MIPv6 [48], and hence the name hierarchical. Moreover, the schemes in this class focus on Intra RO. The schemes (except [18], [25], [46], [47]) have the disadvantage of packets going through one or two tunnels, resulting in near optimal route and header overhead.

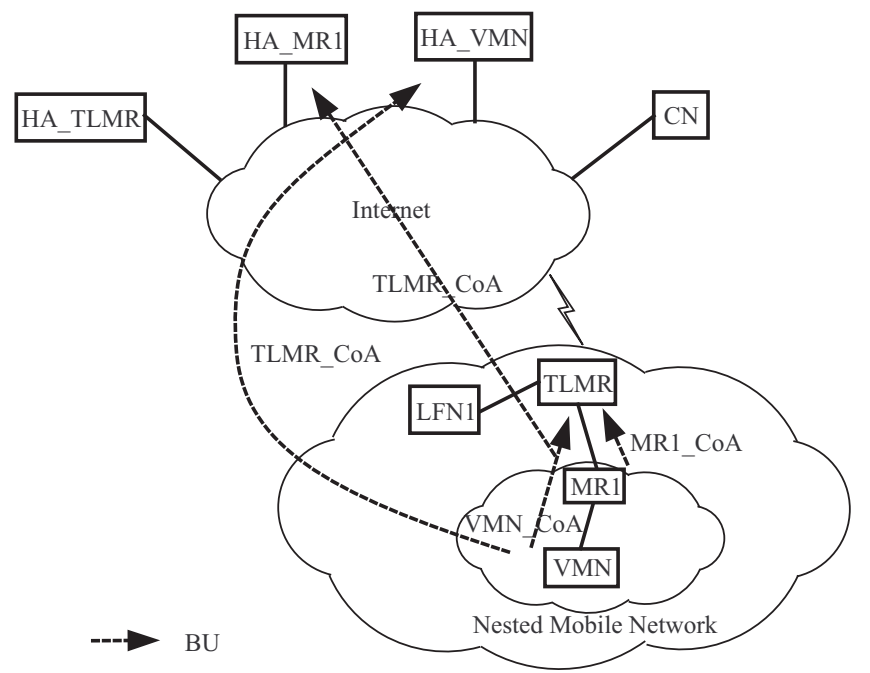

Fig. 5. Hierarchical approach for route optimization.

a) Optimized NEMO (ONEMO): This scheme, proposed by Watari et al. [12], uses extended RA message to convey the CoA of the TLMR to nested MRs. Unlike other schemes in this class, instead of sending the CoA to HA, nested MRs send the $\mathrm{CoA}$ to the nearest router of the $\mathrm{CN}$ that tunnels packets (sent by $\mathrm{CN}$ ) to TLMR. The router that keeps track of the MR's location is discovered by the MR when it receives the first packet through HA. Discovery is initiated by the MR by sending a message to an anycast address which is formed from the prefix of the $\mathrm{CN}$. Discovery of the router and sending $\mathrm{CoA}$ to it require additional signaling; we thus consider it as medium (instead of low) signaling in Table III. The router tunnels the packets, sent by $\mathrm{CN}$ to $\mathrm{MNN}$, to TLMR that route the packets inside the mobile network. Unlike most of the schemes in this class, deployability of the scheme is difficult due to the requirement of support from a router in each CN's network.

b) Route Optimization using Tree Information Option (ROTIO): In ROTIO, proposed by Cho et al. [13], HoA of the TLMR and CoA of intermediate MRs (MRs between the TLMR and an MNN) are conveyed to the MR using RA messages that contains tree information option representing the nesting structure. Each MR appends its CoA to the RA sent by TLMR, and relays the RA down the nesting level. Thus, an MR knows the CoA of intermediate MRs from the $\mathrm{RA}$, and sends two BU: one to its HA containing HoA of TLMR, and another to the TLMR containing list of CoAs of MRs above. Therefore, MR's location is tracked through the HA of the MR, TLMR's HA and the TLMR, and HA of an MR can tunnel packets to TLMR's HoA. TLMR, knowing the nesting structure of the mobile network from the BUs, can route packets inside the mobile network. The disadvantage of this scheme is packets going through two tunnels- one between MR's HA and TLMR's HA, and another between TLMR's HA and TLMR. One additional tunnel as compared to ONEMO is compensated by lower signaling at TLMR's handoff.

c) RO for nested mobile network in local mobility domain using local mobility anchors (LRO): In LRO proposed by Li-hua et al. [14], a prefix used in local mobility domain 
TABLE III

A COMPARISON OF THE RO SCHEMES IN HIERARCHICAL CLASS

\begin{tabular}{|c|c|c|c|c|c|}
\hline Scheme & Degree of RO & Tunnels & Signaling & Memory requirement & Location management \\
\hline ONEMO & Near optimal & One & Medium & Low & HA and Router in CN's network \\
\hline ROTIO & Near optimal & Two & Lower & Low & HA, TLMR's HA and TLMR \\
\hline LRO & Near optimal & One & Low & Low & HA and LMA \\
\hline$\chi$ LIN6-NEMO & Near optimal & None & High & High & Mapping agent \\
\hline HMNR & Near optimal & One & Medium & Low & HA and TLMR \\
\hline ROAD & Optimal & None & High & Low & $\mathrm{HA}$ and $\mathrm{CN}$ \\
\hline HMSRO & Near optimal & One & Low & Low & HA and TLMR or AR \\
\hline Light-NEMO & Near optimal & One & Low & Low & HA and TLMR \\
\hline $\begin{array}{l}\text { Light-NEMO ex- } \\
\text { tended }\end{array}$ & Optimal & None & High & High & $\mathrm{HA}$ and $\mathrm{CN}$ \\
\hline ROPIO & Near optimal & One & Medium & Low & HA and TLMR \\
\hline HMNB & Near optimal & Two & Lower & Low & HA, TLMR's HA and TLMR \\
\hline HIP-based & Optimal & None & High & High & TLMR and CN \\
\hline HMIP-based & Near optimal & Two /three & Low & Low & HA and MAP \\
\hline MoRaRo & Optimal & None & High & High & TLMR and CN \\
\hline
\end{tabular}

is advertised to all MRs through extended RA. MRs obtain $\mathrm{CoA}$ from the prefix, and send $\mathrm{BU}$ to its HA. Another BU sent to Local Mobility Anchor (LMA) contains entries such as CoA, HoA, MRs home prefix and address of HA; therefore, LMA create a BE containing the entries, and performs location management along with HA. A packet, sent from CN to LFN, reaches LFN's HA that tunnels the packet to MR. The packet reaches the LMA that search the destination $(\mathrm{CoA})$ in $\mathrm{BE}$. On finding the address in BE, LMA forwards the packet to MR through intermediate MRs and routers that already have routing entry (created from BU sent by MR to LMA) for the CoA. On reception of packets from local domain, LMA decapsulates the packet to search its BE for the prefix of the inner destination. If found then the packet is routed within the local domain; otherwise, the encapsulation of the packet is restored again and forwarded to HA. Therefore, the scheme can handle Intra RO in a near optimal way but involves one tunneling in all cases.

d) NEMO protocol based on Location Independent Networking in IPv6 ( $\chi$ LIN6-NEMO): In $\chi$ LIN6-NEMO proposed by Banno et al. [15], [16], like ONEMO, MRs obtain the prefix of the foreign network through extended RA, and send the prefix (through $\mathrm{BU}$ ) to the Mapping Agent (MAs) which acts (e.g. performs location management) like the HA. MAs intercept the packets that are sent by $\mathrm{CN}$ to $\mathrm{MNN}$, replace the prefix of the destination address with the prefix of the foreign network, and forwards the packets to the MNN. Unlike other schemes in this class, packets reach TLMR through MA (therefore, near optimal route) without any tunnel because of prefix replacement procedure. TLMR forwards the packet inside the mobile network after restoring the prefix of the destination address to MNN's prefix. Location Independent Networking is achieved by always using the prefix of the network at MNN's current location. Translation of prefix is transparent to the transport layer or above where a location independent address, formed by combining a location independent identifier and a prefix, is used. The scheme decreases the chance of single point of failure by employing multiple MAs dispersed in the Internet resulting in increased signaing required to update all MAs. Moreover, memory requirement is high due to TLMR's tracking of MNNs' prefix to forward packets inside the mobile network. e) Hierarchical Mobile Network Routing (HMNR): In HMNR proposed by Jeong et al. [49], like ONEMO [12], extended RA is used to convey TLMR's CoA to MRs. MCNs send BU containing TLMR's CoA to respective HAs (unlike ONEMO where TLMR's CoA is sent to a router). Therefore, both HA and TLMR in combination keeps track of the MNN's location. Packets sent from $\mathrm{CN}$ to MNN reach HA that tunnels the packets to TLMR. To route packets from TLMR to MNN, each MR maintains a routing table that maps the prefix of an MR to the next hop address. The table is constructed by MRs from the BUs sent from MRs below. Memory requirement for the table is low because number of MRs is small in a mobile network. But, BUs sent from MRs below in addition to BUs sent to HAs results in signaling amount which is a little higher than LRO. Kim et al. [17] proposed another scheme which is similar to HMNR in terms of conveying TLMR's CoA to MRs for sending to their HAs. Unlike HMNR where CoA is obtained from the prefix of the mobile network's home prefix, all MRs obtain CoA from prefix of TLMR, and a routing protocol, preferably RIPng [50], is used to route packets inside mobile networks.

f) Route Optimization using Additional Destinationinformation (ROAD): ROAD, proposed by Park et al. [18], is very similar to HMNR except that this scheme proposes MCNs to send BU, containing TLMR's CoA, to CNs that use the CoA as destination address of packets sent to the MNN. BU also contains MCN's HoA and CoA that are put into an additional header of packets sent by $\mathrm{CN}$. Each MR has a prefix-CoA (of lower level MRs) mapping that is used to overwrite the destination and source of incoming and outgoing packets, respectively. Unlike most of the schemes of this class, this scheme avoids tunneling packets through the HA to secure optimal route at the cost of increased signaling from sending $\mathrm{BU}$ to $\mathrm{CNs}$.

g) Hierarchical Mobility Support for Route Optimization (HMSRO): Kuo et al. [19] proposed HMSRO which is very similar to the HMNR scheme except the routing table construction process. Unlike HMNR, an MR constructs the routing table using the BUs, sent by MRs to TLMR, resulting in fewer number of BUs (i.e. signaling) as compared to HMNR. A scheme proposed by Kim et al. [51] is similar to HMSRO with three exceptions - AR's address is used instead 
of TLMR's CoA, source routing is used to route packet inside the mobile network, and MCNs can send BU to CNs resulting in increase of signaling.

h) Light-NEMO: Light-NEMO, proposed by Jouaber et al. [47], is similar to HMSRO except creation of a routing entry by intermediate MRs from BU sent by an MCN to its HA. Moreover, each MR swaps the source address by its own CoA; eventually HA gets the TLMR's CoA which is used by the HA to tunnel packets to the mobile network. LightNEMO is extended by to remove the HA-TLMR tunnel by having MCNs performing MIPv6 RO, and like MIRON, MRs performing RO on behalf on LFNs [52]. Therefore, unlike most of the schemes in this class, signaling and memory requirement is high. Unlike HMSRO, in extended LightNEMO, CN performs location management along with HA.

i) Optimization using Prefix Information Option (RO$P I O)$ : In ROPIO, proposed by Lu et al. [20], TLMR's prefix and $\mathrm{CoA}$ are advertised (using PIO) to nested MRs that obtain CoAs from TLMR's prefix. Nested MRs send one BU containing its CoA to TLMR, and another containing TLMR'S CoA to HA. Thus, HA and TLMR in combination keeps track of MR's location. Packets sent from $\mathrm{CN}$ reaches HA that tunnels packets to TLMR. TLMR decapsulates and tunnels the packet to the nested MR. Packets on the reverse path are tunneled to HA by MR, and are decapsulated by TLMR that checks if the destination prefix is registered with it. If yes then the packet is tunneled to the MR corresponding to the registered prefix (Intra RO). Otherwise, the packet is tunneled to the HA with with source address changed to TLMR's CoA. The scheme is similar to HMSRO except the process of conveying TLMR's CoA to nested MRs, and therefore, signaling and memory requirement are similar to HMSRO.

j) Hierarchical Mobile Network Binding (HMNB): Jeong et al. proposed HMNB [21] which is similar to HMNR and HMSRO with the exception that in HMNB, like ROTIO, MRs send TLMR's HoA (instead of CoA) to respective HAs. Thus, MRs don't need to send any BU when the TLMR changes network, resulting in less signaling. Disadvantage of this scheme is packets' traversal through two tunnels in contrast to one in HMNR and HMSRO. Depending on the handoff frequency, the scheme in [53] proposes to switch between HMNR and HMNB to tradeoff signaling with one additional tunneling.

k) HIP-based: Host Identity Protocol (HIP) [46] that supports mobility and multihoming for hosts is used for NEMO in the scheme proposed by Ylitalo et al. [22]. In HIP, each host uses a unique address at upper layers, and location changes are managed transparently at HIP or lower layers. At the start of communication in HIP, hosts (one may be an MNN) establish a key that is used for location update. Basic principle of HIP-based NEMO is the use of the key to authorize MR to perform location update on behalf of MNNs. Authorization takes place when an MNN joins the mobile network; in nested NEMO, authorization is performed at various level. When a packet is sent from an MNN to $\mathrm{CN}$, each MR uses prefix translation of the source address to avoid tunneling. Level by level authorization and prefix translation require maintenance of all HIP sessions going through an MR, and hence high amount of memory. Although the scheme does not directly resemble other schemes in the hierarchical class, the scheme is included here because signaling is performed by the TLMR on behalf of all MNNs. Also, like most of the schemes in hierarchical class, TLMR performs location management. Major disadvantages of this scheme are difficulty in wide deployment due to the requirement of HIP in hosts, high signaling to update $\mathrm{CNs}$, and high memory requirement for TLMR to maintain the states for all HIP sessions going through it.

l) HMIP-based: RO based on HMIPv6 [48] is proposed in [54] where MCNs obtains two CoAs - a Regional CoA (RCoA) obtained from the prefix of the Mobility Anchor Point (MAP), and a Local CoA (LCoA) obtained from the prefix of the mobile network. MCNs also send two BUs - one to MAP and another to HA. MAP creates BE from the BU that contains RCoA, LCoA and prefixes of MR, and also extracts the tree structure (used for routing header) of the mobile network. HA creates a BE from the BU containing HoA and RCoA. Thus, $\mathrm{HA}$ and MAP keeps track of an MCN.

Packets, sent from $\mathrm{CN}$ to MNNs, reach HA that tunnels the packets towards MAP using RCoA; MAP uses LCoA to tunnel packets towards the mobile network along with specification of route inside the mobile network using routing header. Packets sent from mobile network to $\mathrm{CN}$ are tunneled by each intermediate MR to avoid ingress filtering, and MAP detunnels the packets before forwarding to $\mathrm{CN}$. Unlike other schemes in this class, MAP performs the RO functionalities of TLMR to avoid sending BU to HA for movement under same domain, incurring low signaling but at the cost of additional tunnels.

The scheme proposed by Kim et al. [55] differs from the scheme proposed in [54] in routing packets inside mobile network. Packets sent from MNN to CN are tunneled by the MR to its HA using RCoA instead of LCoA as the source to avoid tunneling.

Schemes proposed by Park et al. [23] and $\mathrm{Hu}$ et al. [24] extend HMIP-based scheme to reduce BU when the mobile network moves out of MAP's domain, and also avoids tunneling that is required to avoid ingress filtering. Extended scheme proposes that VMNs and LMNs send LCoA (instead of RCoA) to $\mathrm{HA}$ in $\mathrm{BU}$ resulting no $\mathrm{BU}$ when mobile network moves under a different MAP.

m) Mobile router-assisted route optimization for NEMO (MoRaRo): In MoRaRo, proposed by Kafle et al. [25], after receiving the first packet through HA, an MCN sends TLMR's CoA to CNs. The MCN also sends its HoA, CoA, and CN's address to TLMR that creates a binding cache used for routing packets to MNNs, and to send BUs to CNs on behalf of MNNs. Therefore, $\mathrm{CN}$ along with the TLMR performs location management for MNNs. Each MR registers to the MR attached above with its prefix and all prefixes that are reachable through it, and thus MRs are able to route packets inside the mobile network.

Unlike most of the schemes (except HIP-based and ROAD) in this class, $\mathrm{CN}$ can send packets to MNNs without tunneling using TLMR's CoA but at the cost of increased signaling that results from sending $\mathrm{BU}$ to $\mathrm{CNs}$. HoA of MNN is put into an additional header in the packet, and used by TLMR to tunnel the packet to the MNN. Like MIRON, the scheme 


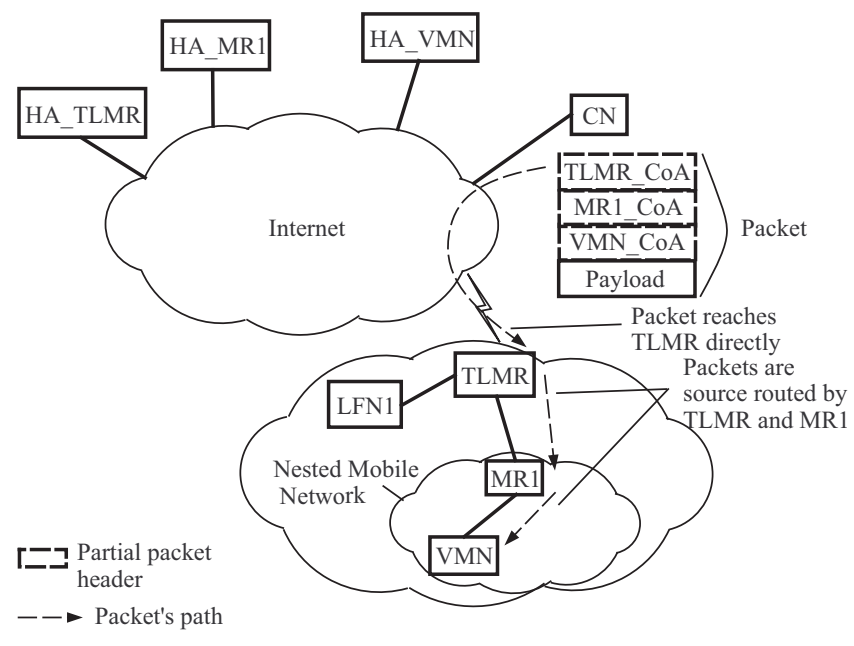

Fig. 6. Source routing approach.

also proposes RO for LFNs by having MRs acting as proxy resulting in high memory requirement for tracking LFN-CN communications.

3) Source routing: In this class, $\mathrm{RO}$ is achieved by sending the CoAs of MRs to the $\mathrm{CN}$ which, like source routing, inserts the CoAs in the packet header to reflect the nesting structure of the MRs. This however, results in increased header overhead. Packets from the CN reach TLMR in an optimal route (without going through HAs); routing within the mobile network is done using the CoAs in the packet header. Memory requirement for routing entries is low because each MR needs to keep track of only the attached MRs as nexthop. Schemes in this class notify $\mathrm{CN}$ about the CoAs of MRs in various ways that will be detailed in the descriptions of the schemes. Notification of CoAs to CNs sacrifices location transparency and deployability, and increases signaling. Methods of notifying the $\mathrm{CN}$ result in differences in signaling and overheads. Moreover, the schemes also have different memory requirement for routing packets inside the mobile network, as shown in Table IV.

Fig. 6 shows the basic principle of the source routing approach where CoAs of TLMR, MR1 and VMN are inserted in packets. Packets, on reaching TLMR, are source routed (using the CoAs) inside the mobile network by TLMR and MR1.

a) Simple Route Optimization (S-RO): In S-RO, proposed by Kim et al. [56], initially the MRs send their CoAs to their respective HAs. Packets sent from $\mathrm{CN}$ are thus encapsulated by the HAs; MRs decapsulate the packets, and send BUs to the source of decapsulated packets. $\mathrm{CN}$ then obtains CoAs of MRs, and sends packets directly to the TLMR with the list of CoAs in the packet header. This scheme suffers from a large delay for the $\mathrm{CN}$ to receive all the CoAs for complete route optimization; this is especially true for higher nesting level. Sending of BUs to HAs and CN results in large amount of signaling.

b) XMIPv6: In xMIPv6, proposed by $\mathrm{Gu}$ et al. [57], MRs send BUs containing CoAs of MRs above it to their corresponding HAs. An MR obtains CoAs of MRs above it from the MR to which it is attached. Packets sent from the $\mathrm{CN}$ to an MNN reach the HA that inserts the CoAs in the header of packets. Unlike S-RO, xMIPv6 does not need BU from all MRs, resulting in the advantage of reduced signaling and smaller time for HA to get CoAs of all MRs above. Unlike other schemes in this class, packets will always go through a tunnel between an HA and the corresponding MR.

c) Path Control Header (PCH)-based: $\mathrm{Na}$ et al. [58] proposed a scheme where the CoA of the MR is inserted into packets by the corresponding HA when a packet travels from an MNN to a CN. After passing through all the HAs, the packet's header contains the CoAs of all MRs above. Path control is achieved by a specific router (between last HA and $\mathrm{CN}$ ) that extracts the CoAs to insert in the packet's header sent from $\mathrm{CN}$ to MNN. Like xMIPv6, this scheme has the advantage of low signaling because of absence of $\mathrm{BU}$ from MRs.

d) Session Initiation Protocol (SIP)-based: Huang et al. [26], [27] proposed a SIP-based [59] RO which, unlike other schemes, uses SIP session establishment procedure to discover an optimized route prior to the start of data communication. An MNN (SIP client) sends a SIP invite request to CN (SIP client) to establish a session. SIP Home Server (acting like HA) inserts CoAs of corresponding SIP-network mobility servers (acting like MRs) into the invite request that reaches $\mathrm{CN}$ with CoAs of network mobility servers. $\mathrm{RO}$ is achieved by $\mathrm{CN}$ inserting the CoAs of network mobility servers in the packets sent to the MNN. At handoff, the SIP-Network mobility server at the top sends invite request (through a SIPforeign server ) to all CNs on behalf of MNNs. Sending invite requests results in high volume of signaling as well as high memory requirement due to the tracking of all SIP sessions.

4) BGP-assisted: Unlike the the schemes described so far, the schemes in this class rely on BGP [60] for mobility management. When the mobile network moves, BGP routers are updated to make necessary changes in the routing tables by making forwarding entries for the prefix of the mobile network. Information regarding the change of route of the mobile network is signaled to few routers that exchange the information with peers using existing routing protocols in the Internet. Therefore, routers contains routing entries to route packets to the mobile network irrespective of its location, and are responsible for location management. Schemes in this class mainly differ (see Table V) in the number of external BGP updates generated, and incurring other overheads for managing Intra RO.

An abstract view of the approach used in this class has been shown in Fig. 7. When the TLMR joins the AR in the foreign network, AR injects a BGP update that maps TLMR's prefix $(1: 3: 1::)$ to AR's address $(1:: 2)$. BGP router3 in AR's network updates its peers (BGP router1 and BGP router2), accordingly. Therefore, packets sent by $\mathrm{CN}$ will reach a BGP router in its network, and will be forwarded to the appropriate BGP router's network where the mobile network resides.

The major advantage of the schemes in this class is the use of no new entity for mobility management. Moreover, CNs are transparent to the change location (managed by BGP routers) of the MNNs. On the other hand, these schemes will produce a storm of updates (i.e. signaling) in the Internet when the mobile network moves frequently. Moreover, scalability is also an issue due to maintenance of routing entries for a large 
TABLE IV

A COMPARISON OF THE RO SCHEMES IN SOURCE ROUTING CLASS

\begin{tabular}{|l|l|l|l|}
\hline Scheme & Signaling & Memory requirement & Other overheads \\
\hline S-RO & High & Low & Large delay to converge to optimized route \\
\hline XMIPv6 & Low & Low & One tunnel is required for communication \\
\hline PCH-based & Low & Low & Requires a router in every network to support the protocol \\
\hline SIP-based & High & High & None \\
\hline
\end{tabular}

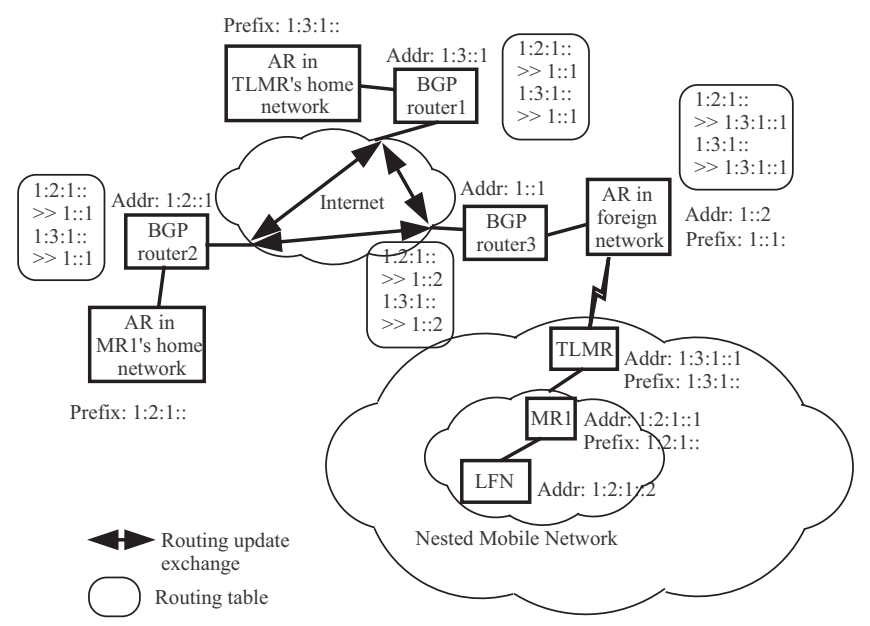

Fig. 7. BGP-assisted approach.

number of mobile networks. Storm of update and scalability has been traded of (i.e. reduced signaling with increased scalability) with some support from the infrastructure resulting in difficulty of deployment. This trade of also requires packets always traveling through one or more of some designated routers resulting in near optimal route.

a) Cellular Universal IP for nested network mobility (CUIP-NEMO): CUIP-NEMO, proposed by Lam et al. [28], is based on Cellular Universal IP (CUIP) [61] where universal addresses are used for a set of mobile nodes that are assumed to be in the same hierarchy of network irrespective of their location. The hierarchy is rooted at a BGP router of the provider network (home network) of mobile nodes, and these routers are directly linked at network layer. Between movements of the node there is a Cross-Over Router (COR) that is the first router in the hierarchy common in both previous and current route. All routers upto COR is updated with the new route of the mobile node using CUIP signaling. A packet sent to the mobile node is routed towards the $\mathrm{CN}$ 's nearest BGP router that routes the packet towards the BGP router closest to the COR. Routers before COR use prefix-based routing whereas routers after COR use inefficient flat routing.

The hierarchical routing structure for host mobility [61] is adapted for mobile networks [28] where ARs are considered as BGP routers, and all MRs are assumed CUIP-enabled routers and hosts. MNNs need not to be aware of CUIP as their packets are handled by MRs. Although this scheme has the advantages of its class, it suffers from the problem of generating frequent updates for routers. The problems will continue to increase with increasing distance of the mobile network from its home network. Moreover, additional signaling is required to discover a COR. b) Wide-Area IP Network Mobility (WINMO): Use of BGP for network mobility is proposed by Dul [29] where the AR, upon attachment of a mobile network, initiates a BGP update announcing the prefix of the mobile network in the Internet. But this may result large routing tables and large number of update messages because of movement of a large number of mobile networks. To limit the routing table size and number of updates, concept of mobile prefixes and aggregation routers are introduced in WINMO proposed by $\mathrm{Hu}$ et al. [30].

A mobile prefix is used to serve all the mobile networks originated from a particular home network. Mobile prefixes are advertised only by a set of routers called aggregation routers that keeps track of sub-prefixes assigned to the mobile networks. Other routers set the closest aggregation router as next hops for mobile prefixes. Whenever a mobile network attaches to a new network, a BGP update is injected to announce the prefix of the mobile network. An aggregation router in the new network shares this update with all other aggregation routers. A packet sent to the mobile network reaches a router that forwards the packet to the closest aggregation router. Aggregation router forwards the packet to the appropriate aggregation router in the network to which the mobile network is attached.

For Intra RO, a mobile router obtains a CoA along with performing authentication with the AR. The CoA is used only to route packet efficiently within the network. A packet sent from the mobile network to $\mathrm{CN}$ carries a key which is generated during authentication by encrypting the prefix of the mobile network and the CoA. The key is managed among all the BGP routers and some other additional routers. Route optimization starts when the reply packet containing the key enters the network. A BGP router checks the validity of the key, and forwards the packet after changing the destination address to the CoA decrypted from the key.

Although this scheme involves smaller number of routers, and generate smaller number of routing updates, it requires changes in BGP. Moreover, involvement of a small group of router for mobility management means route may not be completely optimized all the time. If $\mathrm{CN}$ is unable to recognize the key, the route may not also be completely optimized.

c) Multiple P2P connected HA-based RO: Cuevas [31] proposes deploying multiple HAs that know each other's information (e.g. network, IP etc.) using P2P [62]. A mobile network has a home HA; but can register with any HA to meet certain performance criteria such as a limit for round trip time. To find a closer HA, an MR sends a special BU to its home HA that responds with a list of HAs closer to current location of the mobile network in terms of the performance criteria. MR selects an HA, obtains an HoA, and registers with the selected HA. After registration, HA initiates a BGP update among routers within the network to install the mapping of HoA 
TABLE V

A COMPARISON OF THE RO SCHEMES IN BGP-ASSISTED CLASS

\begin{tabular}{|l|l|l|}
\hline Scheme & Number of external BGP updates & Other overheads \\
\hline CUIP-NEMO & Medium & Signaling to discover COR \\
\hline WINMO & Low & Overhead of key management among routers \\
\hline Multiple P2P connected HA-based & None & $\begin{array}{l}\text { Signaling for P2P communication among HAs, and for dis- } \\
\text { covery of the closest HoA }\end{array}$ \\
\hline
\end{tabular}

to CoA. These routers tunnels/de-tunnels the packet to/from the mobile networks. Change of HA takes place only when a mobile network moves out of the current network, and when an MR finds forwarding through current HA's network is not delivering required performance.

Unlike other schemes in this class, this scheme does not require BGP update outside the network to which the mobile network is attached. But this requires communication, initiated outside, to take place through home network resulting in unoptimized route. In addition, large number of mobile networks that are moving frequently can trigger frequent BGP updates along with the problem of large number of routing table entries.

5) Miscellaneous: This section includes RO schemes that do not fall into any of the previous classes described in Secs. III-C1 - III-C4. The techniques, used for RO in the schemes presented in this section, are different than the basic techniques used for RO in the classes presented in Secs. III-C1 - III-C4. A comparison of the schemes is presented in Table VI.

a) Optimized Route Cache (ORC)-based: Wakikawa [63] et al. proposed an approach where the MR sends BU to a router in the CN's network, and to the MR attached above (parent MR). Parent MR sends a BU, that maps mobile network prefix of the MR underneath (child MR) to parent MR's CoA, to the router performing location management. Therefore, packets, sent to the mobile network, are tunneled to parent MR's CoA by the router that caches the optimized route for tunneling as long as $\mathrm{CN}$ communicates with the MNN. Packets, destined to child MR's network, are decapsulated and forwarded by parent MR to the child MR. To route packets when no router in the CNs' network has the mapping, a BU is sent to a router which is in the home network of the mobile network. MRs uses a routing protocol to route packets inside mobile network.

ORC-based scheme incurs medium (instead of being high) signaling because all MRs send BUs to routers (instead to all $\mathrm{CNs}$ ) in CNs network and to parent MRs. Memory requirement is also high because of maintaining routing entries for all MNNs. A major disadvantage of ORC is that it optimizes route for only one level of nesting. Although route from CNs to MNNs is similar to that in hierarchical class for one level of nesting, it is different when the nesting level increases. In addition, unlike the schemes in hierarchical class, TLMR's HoA or CoA is not conveyed to the nested MRs. Therefore, we have placed this scheme separately in this section.

b) Recursive $B U$ (RBU)-based: Cho et al. [64] proposed a RO scheme where BUs, sent by MRs to $\mathrm{CN}$, are used to recursively process the binding table at $\mathrm{CN}$ to maintain a route to TLMR. On reception of a BU having an HoA which is the same as any of the CoAs in the binding table, the CoA in the table is replaced by the received CoA. CN will eventually have a mapping of MR's prefix to TLMR's CoA after receiving BUs from all MRs, thereby enabling sending of packets directly to TLMR.

Packets are routed inside the mobile network by MRs maintain a routing table, or by TLMR broadcasting a route request for route discovery. Memory requirement for routing will be low when routes are discovered dynamically. Signaling in this scheme is high because MCN and MRs between MCN and AR will send BU to CN. Also, it is not specified how the MRs will know about the $\mathrm{CN}$. This scheme resembles schemes in hierarchical class with the difference of conveying TLMR's HoA or CoA to nested MRs.

c) AODV-based: In AODV-based scheme, proposed by Phang et al. [32], the route between a HA and an MR is established using AODV protocol. After obtaining a CoA from the attached (above) MR's prefix, MR uses AODV route request messages to find a route towards its HA. During this route finding process, all MRs between TLMR and the MR installs the routing entries for routing between the HA and the MR. After route reply is received from the HA, MR sends a $\mathrm{BU}$ to the HA. Packets sent from a $\mathrm{CN}$ first reaches the HA that tunnels the packet to the MR. Since the route from HA to MR is already established by AODV, the packet reaches the MR directly without any further tunneling.

The scheme appears to be very simple; yet, it requires all routers in the Internet, and HA to support AODV resulting the scheme difficult to deploy. Moreover, the scheme involves one tunnel for communication along with overhead of burst of messages (i.e. high signaling) in the Internet during handoff due to broadcast of AODV messages. Although AODV is a protocol for Ad hoc networks, we do not include AODV-based scheme in delegation class under Ad hoc-based scheme due to the following reason. The basic principle used in Ad hoc-based scheme is to obtain a CoA from the foreign network prefix contrasting the obtaining of CoA from MR's prefix in the AODV-based scheme. The scheme also resembles the schemes in hierarchical class in terms of the route except the difference in establishing the route.

\section{Future Research Directions}

Although a considerable amount of research has been carried out in NEMO, we have identified the following issues for future research on NEMO RO:

- Research is required to determine the performance of the RO schemes with change in network topology, i.e. change in the nesting structure of the nested mobile network. Changes in topology can occur in a nested mobile network, formed by mobile networks residing in different vehicles, due to relative movements of the vehicles. Performance analysis of RO schemes needs to be carried out for different mobility patterns. 
TABLE VI

A COMPARISON OF THE RO SCHEMES NOT BELONGING TO ANY PARTICULAR CLASS

\begin{tabular}{|l|l|l|l|l|}
\hline Scheme & Degree of RO & Signaling & Memory requirement & Location management \\
\hline ORC-based & Near optimal & Medium & High & Routers in the Internet \\
\hline RBU-based & Optimal & High & Low & HA and CN \\
\hline AODV-based & Near optimal & High & High & HA \\
\hline
\end{tabular}

- Most of the RO schemes incurs additional signalling over bandwidth limited wireless channels. This contradicts one of the initial objectives of NEMO as a scheme to reduce signalling over wireless channels by letting the mobile router carry out the signalling on behalf of all the nodes. Signaling in RO schemes can be treated in the following ways:

- Update all the CNs and HAs using a single BU. This can be done by letting the CNs and HAs join a multicast group when they join the mobile network.

- RO schemes can be analyzed to find suitable schemes based on the architecture (topology, and number and types of MNNs) of the mobile network, availability of bandwidth, and mobility pattern. For example, when mobile network is moving slowly and has high bandwidth, the schemes that incurs higher signaling to provide complete route optimization for LFNs may be preferable to the schemes that incurs lower signaling at the cost of not optimizing route for LFNs.

- To reduce the signaling, a constant level (one or two) of tunneling can be allowed. This has been done in some of the schemes in hierarchical class, and can be adopted in other schemes dynamically on ad hoc basis.

- Most researchers have worked on RO or improving handoff performance in isolation. However, RO schemes will affect handoff performance due to increased signaling that consumes and competes for bandwidth. To reduce packet loss during handoff, Petander et al. [41] propose the use of multiple interfaces during handoff and evaluated a RO scheme along with the improved handoff procedure. Ryu et al. [65] proposed a handoff scheme where the HA sends packets to the old and new access routers of a mobile router simultaneously. This scheme, however, does not show its performance when RO is used with $\mathrm{CN}$ sending packets directly to MNNs. Therefore, it is important to consider handoff performance along with any RO scheme.

- Security threats also need to be considered in conjunction with RO. Security threats like BU spoofing has been considered for MR-HA [66]. RO allows sending BU to $\mathrm{CNs}$, and makes BU spoofing more vulnerable.

\section{CONCLUSION}

In this paper, we present a survey, classification and comparison among the RO schemes for NEMO. The number of RO schemes reported in this article indicates the exhausting and diverse efforts for RO, and therefore, requires a quantitative evaluation of the RO schemes to determine their suitability and adaptability to the existing Internet infrastructure.
The comparisons show that hierarchical scheme are easier to deploy, and also supports efficient intra mobile network communication at the cost of sacrificing complete optimization of route with the wired network. The implication is that hierarchical schemes may be suitable for mobile networks with higher nesting levels where communications are mainly taking place within the mobile network. A mobile network with no nesting or one/two level of nesting will not benefit from the RO technique of hierarchical approach that trades off allowing one/two tunnels with lower signaling and Intra RO. In addition, most of the communications in the current Internet are client-server type where MNNs are expected to be clients, and servers are the CNs; this also lowers the significance of Intra RO provided by hierarchical schemes. Considering all these, we find that hierarchical schemes are suitable for the networks where hosts inside vehicles form a mobile network, and wants to reach Internet through other vehicles' networks (such as a collection of MANETs).

Delegation-based and BGP-assisted schemes suites the client-server type communication that prevails in the existing Internet. Delegation approach is simple, do not introduce any additional overhead on Internet routing, and optimize route completely; but exerts additional load on the infrastructure due to higher signaling. On the other hand, BGP-assisted approach supports Intra RO, and requires fewer support from infrastructure; but route may not be completely optimized. The relative advantages and disadvantages of these two approaches need to be evaluated to choose one. Source routing approach is not suitable for mobile networks having higher nesting levels due to higher header overhead that consumes bandwidth which is scarce in wireless environment.

The comparison among the schemes within each class reveals the differences among the schemes in more depth. The differences hints the necessity of the evaluation of the schemes under different criteria such as available bandwidth, topology of mobile network, mobility patterns of the mobile network and availability of resources (e.g. memory) in protocol entities. Moreover, signaling and memory requirement depend on the number and types of MNNs in the mobile network, and therefore, might guide the selection of the schemes. Hence, the evaluation under various parameters is required to determine the suitability of the schemes.

To apply the RO schemes to real-world applications and enable their wide deployment, protocol overheads, such as header overhead and signaling need to be reduced. In addition, RO schemes should not require significant changes to the existing Internet infrastructure and protocols (i.e. addressing, functioning of the routers, too many new entities etc.).

\section{REFERENCES}

[1] C. Perkins. IP mobility support for IPv4. RFC 3220, Jan. 2002. 
[2] D. B. Johnson, C. E. Parkins, and J. Arkko. Mobility support in IPv6. RFC 3775, Jun. 2004.

[3] D. Le, X. Fu, and D. Hogrefe. A review of mobility support paradigms for the Internet. IEEE Commun. Surveys Tutorials, 8(1):38-51, First Quarter 2006.

[4] V. Devarapalli, R. Wakikawa, A. Petrescu, and P. Thubert. NEtwork MObility (NEMO) basic support protocol. RFC 3963, Jan. 2005.

[5] A. Conta and S. Deering. Generic packet tunneling in IPv6 specifications. RFC 2473, Dec. 1998.

[6] E. Perera, V. Sivaraman, and A. Seneviratne. Survey on network mobility support. ACM SIGMOBILE Mobile Computing and Communications Review, 8(2):7-19, Apr. 2004.

[7] F. Mimoune, F. Nat-Abdesselam, T. Taleb, and K. Hashimoto. Route optimization for large scale network mobility assisted by BGP. In IEEE GLOBECOM, Washington, DC, USA, Nov. 26-30 2007.

[8] W. Su, H. Zhang, and Y. Ren. Research on route optimization in mobile networks. In International Conference on Wireless Communications, Networking and Mobile Computing, pages 1-4, Wuhan City, China, Sep. 22-24, 2006.

[9] H. Park, T.J. Lee, and H. Choo. Optimized path registration with prefix delegation in nested mobile networks. In International Conference on Mobile Ad-hoc and Sensor Networks, Wuhan, China, Dec. 13-15, 2005.

[10] S. Novaczki, L. Bokor, G. Jeney, and S. Imre. Design and evaluation of a novel HIP-based network mobility protocol. J. Networks, 3(1):10-24, Jan. 2008.

[11] S. Novaczki, L. Bokor, and S. Imre. A HIP based network mobility protocol. In International Symposium on Applications and the Internet, Hiroshima, Japan, Jan. 15-19 2007.

[12] M. Watari, T. Ernst, and J. Murai. Routing optimization for nested mobile networks. IEICE Trans. Commun., E89-B(10):2786-2793, Oct. 2006.

[13] H. Cho, T. Kwon, and Y. Choi. Route optimization using tree information option for nested mobile networks. IEEE J. Sel. Areas Commun. 24(9):1717-1724, Sep. 2006

[14] L. Li-hua, X.L. Ma, and L. Yuan-an. Route optimization solution for nested mobile network in local mobility domain with multiple local mobility anchors. In International Symposium on Communications and Information Technologies, Sydney, Australia, Oct. 16-19 2007.

[15] A. Banno and F. Teraoka. $\chi$ LIN6: An efficient network mobility protocol in IPv6. In International Conference on Information Networking: Advances in Data Communications and Wireless Networks, Sendai, Japan, Jan. 16-19, 2006.

[16] A. Banno, T. Oiwa, and F. Teraoka. $\chi$ LIN6-NEMO: A network mobility protocol based on LIN6. IEICE Trans. Commun., E88-B(7):2765-2776, Jul. 2005.

[17] S. H. Kim, Y. Y. Ahn, S. H. Kim, and T. I. Kim. Route optimization using RIPng protocol in nested network mobility. In 8th International Conference Advanced Communication Technology, Phoenix Park, Korea, Feb. 20-22, 2006

[18] J. Park, S. Lee, Y. Lee, and H. Choo. Route optimization with additional destination-information in mobile networks. In International Conference on Computational Science and Its Applications, Kuala Lumpur, Malayasia, Aug. 26-29, 2007.

[19] G.S. Kuo and K. Ji. Novel hierarchical network mobility support protocol with bidirectional end-to-end route optimization solution for nested mobile networks. In IEEE GLOBECOM, San Francisco, CA, Nov. 27-Dec. 1, 2006.

[20] L.H. Lu and Y.A. Liu. Route optimization solution based on extended prefix information option for nested mobility network. In International Conference on Wireless Communications, Networking and Mobile Computing, Shanghai, P.R. China, Sep. 21-23, 2007.

[21] M. S. Jeong, Y. H. Cho, and J. T. Park. Hierarchical mobile network binding scheme for route optimization in NEMO. Wireless Personal Commun., 43(3):975-995, Nov. 2007.

[22] J. Ylitalo, J. Melen, P. Salmela, and H. Petander. An experimental evaluation of a HIP based network mobility scheme. In International Conference on Wired/Wireless Internet Communications, Tampere, Finland, Mar. 122008.

[23] J. Park, T.J. Lee, and H. Choo. Route optimization with MAP-based enhancement in mobile networks. In International Conference on Computational Science, University of Reading, UK, May 28-31 2007.

[24] J.Y. Hu, C.F. Chou, M.S. Sha, I.C. Chang, and C.Y. Lai. On the design of micro-mobility for mobile network. In IFIP International Conference on Embedded and Ubiquitous Computing, Taipei, Taiwan, Dec. 17-20 2007.

[25] V. P. Kafle, E. Kamioka, and S. Yamada. MoRaRo: Mobile routerassisted route optimization for Network Mobility (NEMO) support. IEICE Trans. Inf. Syst., E89-D(1):158-170, Jan. 2006.
[26] C.M. Huang and C.H. Lee. Signal reduction and local route optimization of SIP-based network mobility. In IEEE Symposium on Computers and Communications, Sardinia, Italy, Jun. 26-29, 2006.

[27] C.M. Huang, C.H. Lee, and J.R. Zheng. A novel SIP based route optimization for network mobility. IEEE J. Sel. Areas Commiun., 24(9):1682-1691, Sep. 2006.

[28] P. Lam, S. Liew, and J. Lee. Cellular universal IP for nested network mobility. Computer Networks, 51(12):3617-3631, Aug. 2007.

[29] A. Dul. Global IP network mobility using border gateway protocol. http://www.quark.net/docs/Global_IP_Network_Mobility _using_BGP.pdf, Mar. 2006.

[30] X. Hu, L. Li, Z. M. Mao, and R. Yang. Wide-area IP network mobility. In IEEE INFOCOM, Arizona, USA, Apr. 13-19, 2008.

[31] R. Cuevas, A. Cabellos-Aparicio, A. Cuevas, J. Domingo-Pascual, and A. Azcorra. fP2P-HN: A P2P-based route optimization architecture for mobile IP-based community networks. Computer Networks, 53(4):528540, Mar. 2009.

[32] S.Y. Phang, H. Lee, and H. Lim. Route optimization for NEMO based on AODV. In International Conference on Advanced Communication Technology, Phoenix Park, Republic of Korea, Feb. 17-20 2008.

[33] H. Lim, D. Lee, T. Kim, and T. Chung. A model and evaluation of route optimization in nested NEMO environment. IEICE Trans. Commun., E88-B(7):2765-2776, Jul. 2005.

[34] T. Ernst and H-Y. Lach. Network mobility support terminology. RFC 4885, Nov. 2007.

[35] C. Ng, P. Thubert, M. Watari, and F. Zhao. Network mobility route optimization problem statement. RFC 4888, Jul. 2007.

[36] C. Ng, F. Zhao, M. Watari, and P. Thubert. Network mobility route optimization solution space analysis. RFC 4889, Jul. 2007.

[37] K.J. Lee, J. Park, and H. Kim. Route optimization for mobile nodes in mobile network based on prefix delegation. In IEEE 58th Vehicular Technology Conference, pages 2035-2038, Orlando, Florida, USA, Oct. 6-9, 2003.

[38] J. Jeong, K. Lee, J. Park, and H. Kim. Route optimization based on NDProxy for mobile nodes in IPv6 mobile network. In IEEE 59th Vehicular Technology Conference, pages 2461 - 2465, Milan, Italy, May 17-19, 2004.

[39] J. Song, S. Han, and K. Park. Route optimization in nemo environment with limited prefix delegation mechanism. In International Conference on Computational Science, University of Reading, UK, May 28-31 2006.

[40] E. Perera, A. Seneviratne, and V. Sivaraman. Optinets: An architecture to enable optimal routing for network mobility. In International Workshop on Wireless Ad-Hoc Networks, pages 68-72, Oulu, Finland, May 31-Jun. 3, 2004.

[41] H. Petander, E. Perera, K.C. Lan, and A. Seneviratne. Measuring and improving the performance of network mobility management in IPv6 networks. IEEE J. Sel. Areas Commiun., 24(9):1671-1681, Sep. 2006.

[42] C. J. Bernardos, M. Bagnulo, and M. Calderon. MIRON: MIPv6 route optimization for NEMO. In 4th Workshop on Applications and Services in Wireless Networks, Boston, Massachusetts, USA, Aug. 8-11, 2004.

[43] M. Calderon, C. J. Bernardos, M. Bagnulo, I. Soto, and A. de la Oliva Design and experimental evaluation of a route optimization solution for NEMO. IEEE J. Sel. Areas Commiun., 24(9):1702-1716, Sep. 2006.

[44] D. Forsberg, Y. Ohba, B. Patil, H. Tschofenig, and A. Yegin. Protocol for carrying authentication for network access (PANA). RFC 5191, May 2008.

[45] M. Kim, H. Radha, and H. Choo. On multicast-based binding update scheme for nemo environments. In The International Conference on Computational Sciences and its Applications, Krakw, Poland, Jun. 23252008.

[46] R. Moskowitz and P. Nikander. Host identity protocol (HIP) architecture. RFC 4423, May 2006.

[47] B. Jouaber M. Sabeur and D. Zeghlache. MR-proxy based solution for nested mobile network problems. In International Symposium on Wireless Personal Multimedia Communications, Aalborg, Denmark, Sep. $17-22,2005$

[48] H. Soliman, C. Castelluccia, K. El Malki, and L. Bellier. Hierarchical mobile IPv6 mobility management (HMIPv6). RFC 4140, Aug. 2005.

[49] M. S. Jeong and J. T. Park. Hierarchical mobile network routing: route optimization and micro-mobility support for NEMO. In Proc. International Conference on Embedded and Ubiquitous Computing, Aizu-wakamatsu, Japan, Aug. 25-27, 2004.

[50] G. Malkin and R. Minnear. RIPng for IPv6. Internet Draft,, Jan. 1997.

[51] Y. Kim, S. Woo, S. Kang, and W. Park. Local source routing based route optimization in nested mobile networks. In International Conference on Computational Science and Its Applications, Glasgow, UK, May 8-11, 2006. 
[52] B. Jouaber M. Sabeur and D. Zeghlache. Light-nemo+: Route optimzation for light-nemo solution. In IEEE International Conference on Networks, Singapore, Sep. 13-15, 2006.

[53] M.S. Jeong, Y.H. Cho, and J.T. Park. Policy-based route optimization for network mobility of next generation wireless networks. In 5th International Conference on Ad-Hoc, Mobile, and Wireless Networks, Ottawa, Canada, Aug. 17-19, 2006

[54] H. Ohnishi, K. Sakitani, and Y. Takagi. HMIP based route optimization method in a mobile network. Internet Draft, Oct. 2003

[55] K. Kim, D. Lee, J. Y. Ahn, and H. H. Lee. Hierarchical route optimization in mobile network and performance evaluation. In Second International Conference on Embedded Software and Systems, Xi'an, P.R. China, Dec. 16-18, 2005.

[56] H. Kim, G. Kim, and C. Kim. S-RO: Simple route optimization scheme with NEMO transparency. In International Conference on Information Networking, pages 401-411, Jeju Island, Korea, Jan. 31-Feb. 2, 2005.

[57] Z. Gu, D. Yang, and C. Kim. Mobile IPv6 extensions to support nested mobile networks. In 18th International Conference on Advanced Information Networking and Application, pages 488-491, Tokyo, Japan, Mar. 29-31, 2004.

[58] J. Na, J. Choi, S. Cho, C. Kim, S. Lee, H. Kang, and C. Koo. A unified route optimization scheme for network mobility. In 9th International Conference on IFIP TC6, pages 29-38, Delft, Netherlands, Sep. 21-23, 2004.

[59] J. Rosenberg et al. SIP: Session initiation protocol. RFC 3261, Jun. 2002.

[60] Y. Rekhter, T. Li, and S. Hares. A Border Gateway Protocol 4 (BGP-4). RFC 4271, Jan. 2006

[61] P. Lam, S. Liew, and J. Lee. Cellular universal IP: A low delay mobility scheme based on universal IP addressing. In The 8th ACM International Symposium on Modeling, Analysis and Simulation of Wireless and Mobile Systems, Montreal, Qc. Canada, Oct. 10-13, 2005.

[62] Peer-to-peer. http://en.wikipedia.org/wiki/Peer-to-peer.

[63] R. Wakikawa, S. Koshiba, K. Uehara, and J. Murai. ORC: Optimize route cache management protocol for network mobility. In 10th International Conference on Telecommunications, pages 1194-1200, Tahiti, French Polynesia, Feb. 23-Mar. 1, 2003.

[64] H. Cho, E. K. Paik, and Y. Choi. RBU+: Recursive binding update for end-to-end route optimization in nested mobile networks. In 7th IEEE International Conference on High Speed Networks and Multimedia Communications, pages 468-478, Toulouse, FRANCE, Jun. 30-Jul. 2, 2004.

[65] H. K. Ryu, D. H. Kim, Y. Z. Cho, K. W. Lee, and H. D. Park Improved handoff scheme for supporting network mobility in nested mobile networks. In International Conference on Computational Science and Its Applications, pages 378-387, Singapore, May 9-12, 2005.

[66] S. Jung, F. Zhao, and H. Kim. Threat analysis on network mobility (NEMO). In Sixth International Conference on Information and Communications Security, Malaga, Spain, Oct. 27-29, 2004.

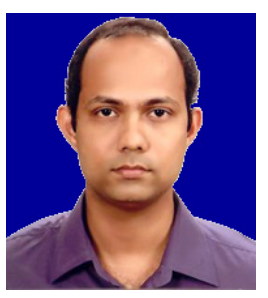

Abu Zafar M. Shahriar Abu Zafar M Shahriar received his BSc and MSc degrees from Bangladesh University of Engineering and Technology, Bangladesh in the year 1999 and 2004 respectively, both in Computer Science and Engineering. Currently he is a Research Assistant and working towards his $\mathrm{PhD}$ in the School of Computer Science at University of Oklahoma. His research interests include mobility of IPv6 networks in terrestrial and space networks, and file transfer protocols for hybrid space-terrestrial networks. He has several conference and journal papers published by IEEE and Springer.

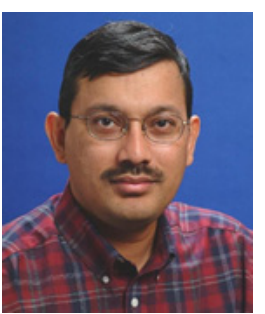

Mohammed Atiquzzaman Mohammed Atiquzzaman obtained his M.S. and Ph.D. in Electrical Engineering and Electronics from the University of Manchester (UK). He is currently a professor in the School of Computer Science at the University of Oklahoma, and a senior member of IEEE.

Dr. Atiquzzaman is the editor-in-chief of Journal of Networks and Computer Applications, co-editorin-chief of Computer Communications journal and serves on the editorial boards of IEEE Communications Magazine, International Journal on Wireless and Optical Communications, Real Time Imaging journal, Journal of Communication Systems, Communication Networks and Distributed Systems and Journal of Sensor Networks. He also guest edited 12 special issues in various journals.

He has served as symposium co-chairs for IEEE Globecom $(2006,2007)$ and IEE ICC $(2007,2009)$ conferences. He co-chaired ChinaComm (2008), and co-chairs for SPIE Next-Generation Communication and Sensor Networks (2006), IEEE High Performance Switching and Routing Symposium (2003) and the SPIE Quality of Service over Next Generation Data Networks conferences $(2001,2002,2003,2005)$. He was the panels co-chair of INFOCOM'05, and is/has been in the program committee of numerous conferences such as INFOCOM, Globecom, ICCCN, Local Computer Networks.

He serves on the review panels of funding agencies such as the National Science Foundation and National Research Council (Canada) and Australian Research Council (Australia). In recognition of his contribution to NASA research, he received the NASA Group Achievement Award for "outstanding work to further NASA Glenn Research Center's effort in the area of Advanced Communications/Air Traffic Management's Fiber Optic Signal Distribution for Aeronautical Communications" project. He is the co-author of the book "Performance of TCP/IP over ATM networks" and has over 220 refereed publications, most of which can be accessed at www.cs.ou.edu/ atiq.

His research interests are in communications switching, transport protocols, wireless and mobile networks, ad hoc networks, satellite networks, Quality of Service, and optical communications. His research has been funded by National Science Foundation (NSF), National Aeronautics and Space Administration (NASA), U.S. Air Force, and Cisco through grants totaling over $\$ 3.8 \mathrm{M}$

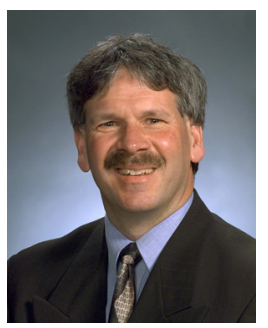

William Ivancic Mr. Ivancic has over twenty-five years experience in network and system engineering for communication applications, communication networking research, state-of-the-art digital, analog and RF hardware design and testing. He currently is a senior research engineer at NASA's Glenn Research Center where he directs the hybrid satellite/terrestrial networking, space-based Internet, and aeronautical Internet research. He has lead research efforts to deploy commercial-off-the-shelf (COTS) technology into NASA missions including the International Space Station and Shuttle. Mr. Ivancic is also performing joint research with Cisco System on advance routing research for space-based and aeronautic-based networks. Of particular interest is large scale, secure deployment of mobile networks including mobile-ip and mobile router technology. Recent accomplishments include: "1st to demonstrate and deploy secure mobile networks in an operational government network, the US Coast Guard " 1st to deploy Mobile-IP Mobile networking on a space-based asset, the Cisco router in Low Earth Orbit (CLEO) "1st to deploy Internet Protocol security (IPsec) and Internet Protocol version 6 on a space-base asset "1st to deploy delay/disruption network technology bundling protocol in space.

Mr. Ivancic is also principle of Syzygy Engineering, a small consulting company specializing in communications systems and networking as well as advanced technology risk assessment. Mr. Ivancic is currently performing research and development on Identity-based security and key and policy management and distribution for tactical networks - particularly mobile networks. 